\title{
Photovoltaic Power System Simulation based on MATLAB/Simulink Software
}

\author{
Guo Yaqin, Song Xin, Dai Chengjun \\ Electrical Engineering and Energy College,Nantong Polytechnic College,Nantong, China \\ guoyq1981@126.com, 1126891682@qq.com, 2732808254, @qq.com
}

\begin{abstract}
Keywords: MATLAB/ Simulink; Photovoltaic Power ; simulation; Solar Panel
Abstract: Photovoltaic power is increasingly concerned in the field of new energy. Photovoltaic modules simulation can provide theoretical basis for photovoltaic power system. In the paper, it is introduced that, construct testing model, draw output curve, set different parameters in the model, and process the output data, then obatin $U-I$ and $P-U$ curves in the different parameters. It was proven that integrated design of photovoltaic power system can be obtained using MATLAB/Simulink software.
\end{abstract}

\section{Introduction}

With the development of global new energy technology, the research for renewable energy is gradually deepened all countries. In order to promote standardization and marketization of new energy industry, a series of development plan is proposed in China. In the energy development strategy plan (2014-2020) ${ }^{[1]}$,it is proposed that installed capacity will reach 58 million kilowatts, and meanwhile the construction installed capacity will reach 30 million kilowatts until 2020. The research shows that photovoltaic power will occupy an important position in the world energy consumption market, and become the subject of the world energy supply. With the support of national policy, researchers are required to provide technical support.

Photovoltaic power system include solar panels, controllers and inverters ${ }^{[2]}$. In the practical application, according to the principle of each part, mathematical model is established to describe its characteristics, and different parameters are set up to find the optimal theoretical method. MATLAB/Simulink simulation software has good modeling environment ${ }^{[3]}$, and the method of photovoltaic power system using MATLAB/Simulink simulation software is proposed in the paper.

\section{Photovoltaic Power Systerm MATLAB/Simulink Simulation Model}

MATLAB/ Simulink simulation software can construct complex mathematical model. Simulation software is convenient and flexible, not limited by place and time, as long as install the appropriate software in the computer, meanwhile researchers can modify the parameters in real time and improve the model ${ }^{[4]}$.

Photovoltaic power system start from the solar panel, take load and power grid as the end, the system is controlled by controller and inverter ${ }^{[5]}$. More contents are included in the photovoltaic power principle, many models can be demonstrated visibly. The simulation model mainly includes: solar panel simulation model, maximum power point tracking simulation model, electric energy conversion circuit simulation model, battery charging and discharging simulation model, inverter photovoltaic grid-connected system and so on. This paper take the construction of the solar panel testing model as a example, introduce in detail the modeling method, set different parameters in the model, place output data into the specified file, and draw $U-I$ and $P-U$ curves using MATLAB commands, then analyze the effects of system parameters on curve. 


\section{Solar Panel Simulation}

Solar Panel Theoretical Analysis.The solar panel is composed of PN junction. The conversion of energy is based on the photovoltaic effect of PN junction. When the battery board accept light, holes and electrons are generated at the ends of the PN junction ${ }^{[6]}$. The either end of the battery board are connected with the load, and the load will have current. The equivalent circuit of the solar panel under light is shown in Figure 1. The circuit is composed of current source, diode and resistors.

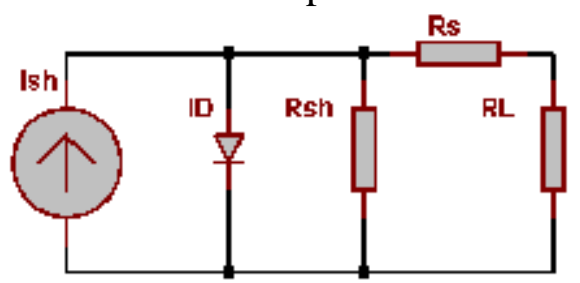

Fig. 1 solar panel circuit diagram under sunlight

Theoretical algorithm ${ }^{[7]}$ of solar panel is obtained from Fig. 1.

$$
\begin{aligned}
& I=I_{s c}\left[1-C_{1}\left(e^{\frac{V-\Delta V}{C_{2} V_{o c}}}-1\right)\right]+\Delta I \\
& C_{1}=\left(1-\frac{\mathrm{I}_{\mathrm{m}}}{I_{s c}}\right) e^{-\frac{V_{m}}{C_{2} V_{o c}}} \\
& C_{2}=\frac{\frac{V_{m}}{V_{o c}}-1}{I n\left(1-\frac{\mathrm{I}_{\mathrm{m}}}{I_{s c}}\right)} \\
& \Delta I=\alpha \frac{R}{R_{r e f}} \Delta T+\left(\frac{R}{R_{r e f}}-1\right) I_{s c} \\
& \Delta V=-\beta \Delta T-R_{s} \Delta I \\
& \Delta T=T_{c}-T_{r}
\end{aligned}
$$

In the formula, $I_{s c}$ and $I_{m}$ is maximum operating point current and short-circuit current, $V_{o c}$ and $V_{m}$ is open-circuit voltage and maximum operating point voltage, $R_{r e f}$ is solar radiation intensity and value is $1 \mathrm{~kW} / \mathrm{m}^{2}, T_{r}$ is solar cell temperature reference value and value is $25^{\circ} C, \alpha$ and $\beta$ is temperature coefficient of current and voltage, $R_{s}$ is array series resistor of solar panel. The parameters in the model are shown in Table 1.

Table 1 model papemeters

\begin{tabular}{|c|c|c|c|c|c|c|}
\hline $\mathrm{V}_{\mathrm{oc}} / \mathrm{V}$ & $\mathrm{V}_{\mathrm{m}} / \mathrm{V}$ & $\mathrm{I}_{\mathrm{sc}} / \mathrm{A}$ & $\mathrm{I}_{\mathrm{m}} / \mathrm{A}$ & $\alpha$ & $\beta$ & $\mathrm{R}_{\mathrm{s}} / \Omega$ \\
\hline 21.6 & 17.2 & 3.41 & 3.19 & 0.015 & -0.07 & 2 \\
\hline
\end{tabular}

Solar Panel Simulation Model. According to the solar panel theoretical analysis, the simulation model is shown in Figure 2. In1 is model input-port, used to set input voltage. If the input voltage is $0 \sim 25 \mathrm{~V}$, input the $\mathrm{t}=[0: 0.1: 25]^{\prime}, \mathrm{u}=\mathrm{t}$ command in the MATLAB command window, and the output waveform can be observed in real time by oscilloscope. In the simulation model, solar cell temperature value $\mathrm{T}$ is $25^{\circ} \mathrm{C}$, sunlight intensity $\mathrm{R}$ is $1 \mathrm{~kW} / \mathrm{m}^{2}$, set parameters in the " Data Import/Export", import data from the workspace into the model, and place output data into the specified file, then call data from workspace files in the command window, draw $U-I$ and $P-U$ curves. The curves are shown in Figure 3 and Figure 4.

Model Analysis. In the simulation experiment, we set different solar panel temperature and sunlight intensity, obtain the experiment data of different parameters, and use the MATLAB 
command to process the output data, and draw curves. It is compared that $U-I$ and $P-U$ curves under different solar panel temperatures in Figure 5 (a) and Figure 5 (b). It is compared that $U-I$ and $P-U$ curves under different sunlight intensity in Figure 6 (a) and Figure 6 (b). From this figure, you can see that, when the solar panel temperature is fixed, and the sunshine intensity rises from $200 \mathrm{~W} / \mathrm{m}^{2}$ to $1000 \mathrm{~W} / \mathrm{m}^{2}$, the maximum operating voltage has not changed much, but the maximum power point increases accordingly. When the sunlight intensity is fixed, and the solar panel temperature rises from $0^{\circ} \mathrm{C}$ to $75^{\circ} \mathrm{C}$, the maximum operating voltage and the maximum power point decrease accordingly. It is obtained that the simulation model results are exactly the same as the theoretical analysis from the experimental results.

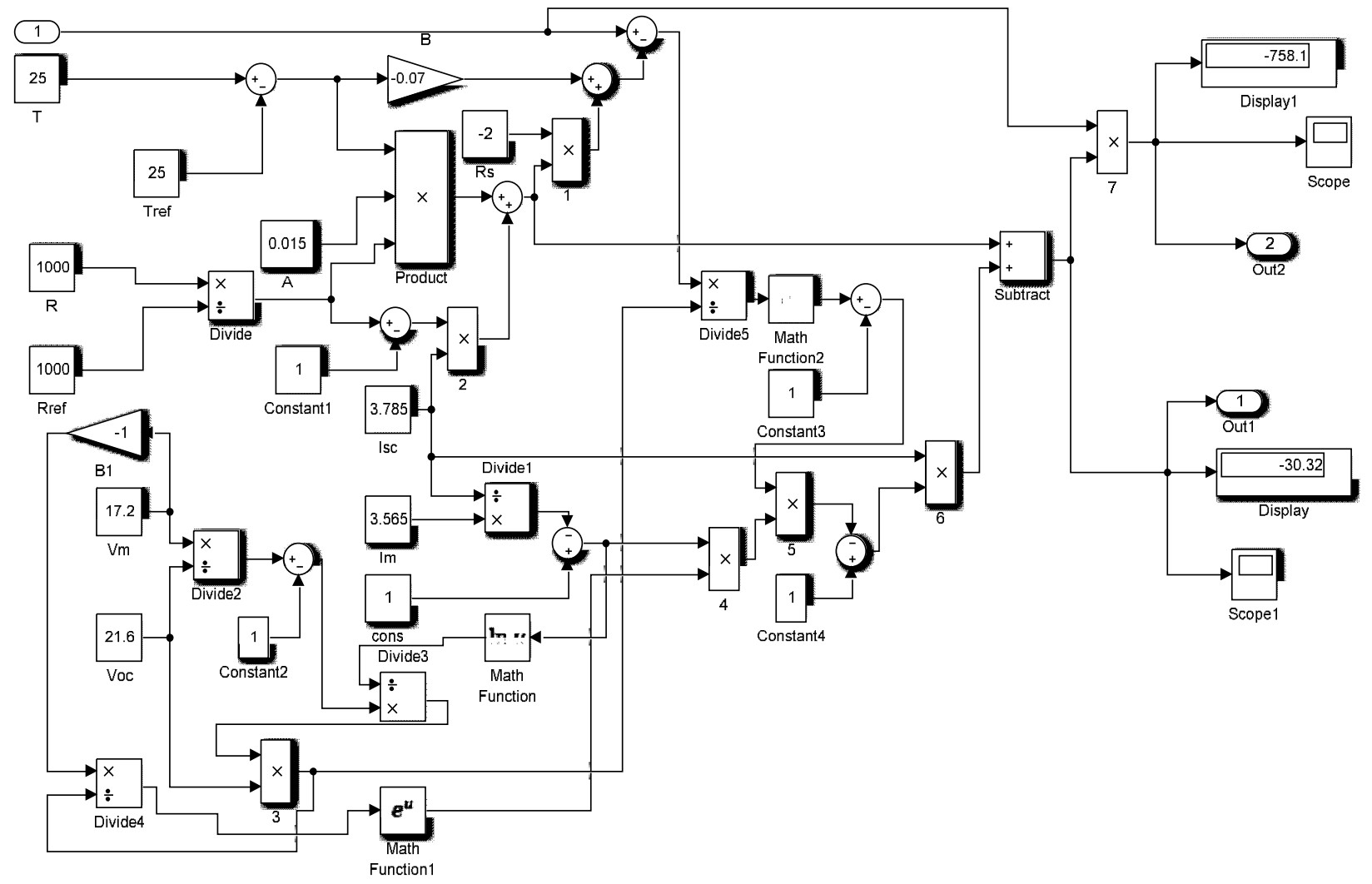

Fig. 2 solar panel model

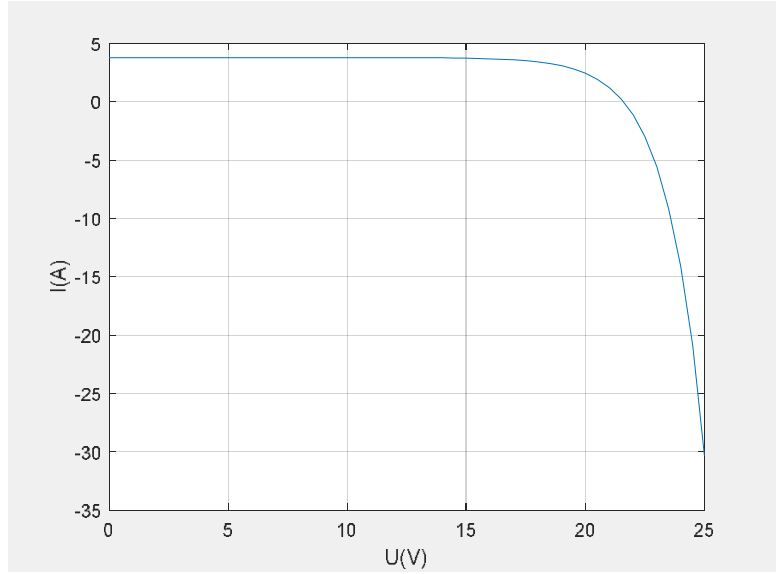

Fig. $3 U-I$ curve

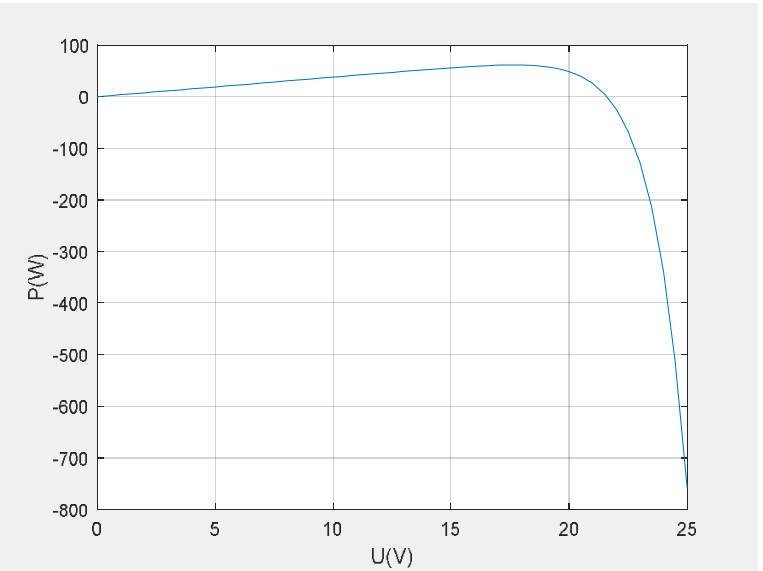

Fig. $4 P-U$ curve

\section{Conclusions}

According to the advantages of MATLAB/ Simulink software modeling and dynamic analysis, we apply the software to photovoltaic power systems. Taking the solar panel modeling as a case, the constructing method of the model and the processing method of the output data are introduced in 
detail. In the model, the experimental results show that the output characteristic of solar panels is described intuitively, and the output results can be processed constantly, thus the output characteristics can be obtained. In the process of experiment, according to self-designed circuit, we can modify conveniently the model and parameters of circuit, and prove feasibility of the circuit. We apply the MATLAB/ Simulink software to the solar photovoltaic power system, providing technical support for the photovoltaic industry, and meanwhile promote the country energy development plan.

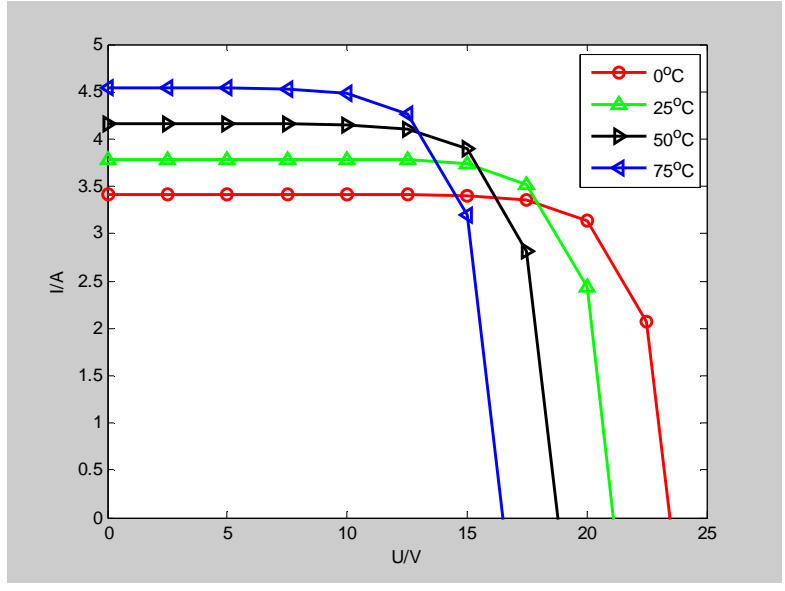

Fig. 5(a) $U-I$ characteristic curve

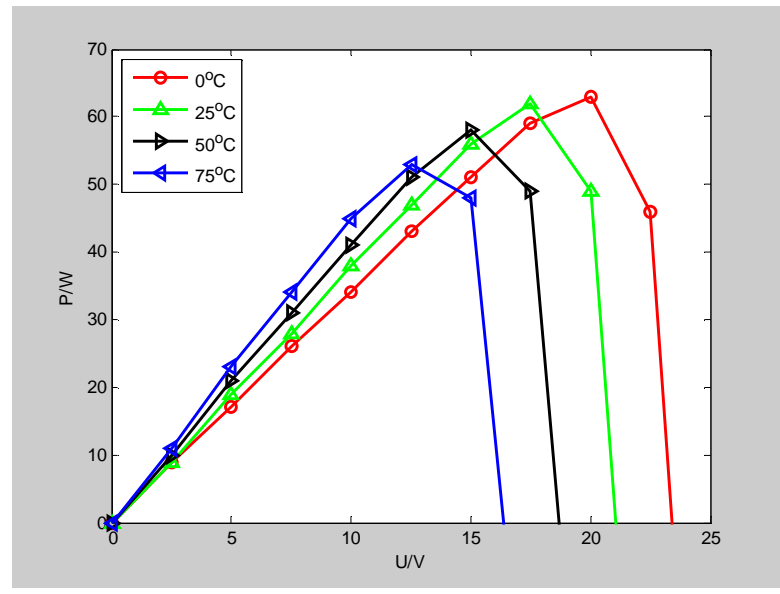

Fig. 5(b) $P-U$ characteristic curve

Fig. 5 characteristic curves of solar panel under temperture

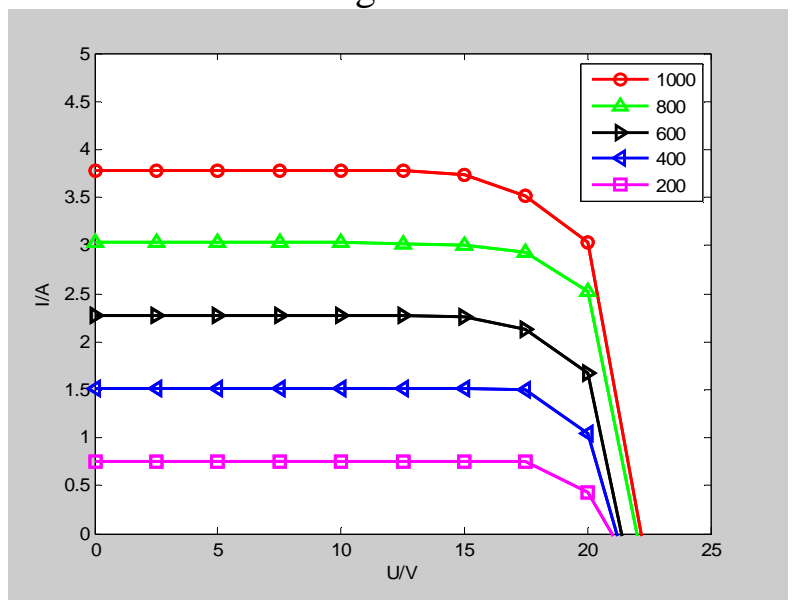

Fig. 6(a) $U-I$ characteristic curve

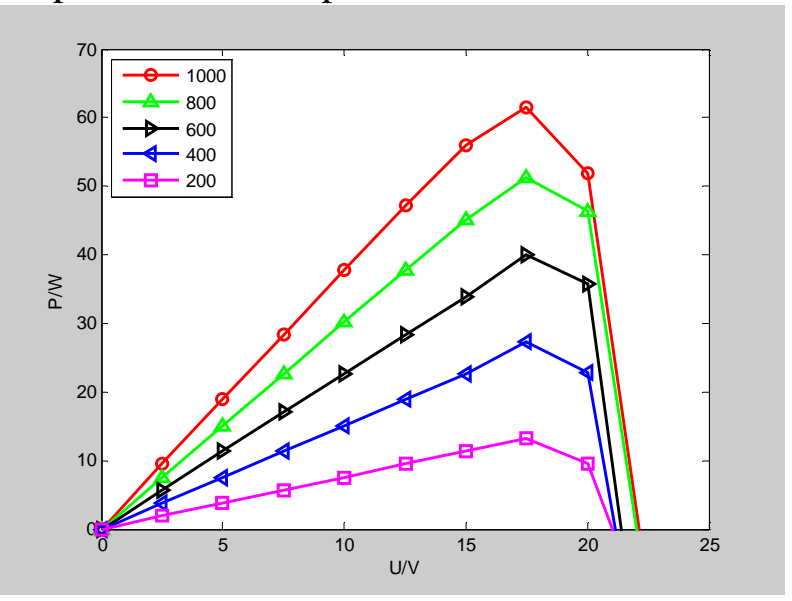

Fig. 6(b) $P-U$ characteristic curve

Fig. 6 characteristic curves of solar panel under sunlight intensity

\section{Acknowledgements}

This work was financially supported by the Jiangsu College Students Innovation Foundation (201712056009Y).

\section{References}

[1] Information on http://www. gov.cn .

[2] ZHAO Liujie, HE Lisan, HAO Qingying. Study on the Test of Distributed Residential Grid-Connected PV Power Generation System. Energy and Energy Conservation.2017(1):63-67.

[3] Qu Bogang,Yi Yingping.Construction and implementation of co-simulation platform based on CCS and MATLAB/Simulink. Computer Technology and Its Applications.2016(4):105-110.

[4] Liu Dongran,Chen Shuyong,Ma Min. A Review on Models for Photovoltaic Generation System. Power System Technology.2011(8):47-52.

[5] Xu Shao-lun, Sun Jia, Xu Qing-jing. Design and Application of Power Quality Comprehensive Experimental Platform. Research and exploration in laboratory. 2015(8):73-76. 
[6] GUO Ya-qin, WANG Zheng-qun,QIN Yan. Application of Proteus in Design and Production of Electronic Products. Research and exploration in laboratory.2013(12):101-104.

[7] Zhang Yu,Mo Han,Zhang Lieping. Photovoltaic power prediction based on fuzzy support vector machine. Thermal power generation.2017(1):116-120. 